\title{
Small Cell Lung cancer: Molecular Oncology Approach
}

\author{
*Zuhair Elkhair Ahmed \\ Department of Medical Oncology, King Abdullah Medical City, Saudi Arabia
}

Submission: June 10, 2017; Published: June 26, 2017

"Correspondence author : Zuhair Elkhair Ahmed, King Abdullah Medical City, Department of Medical Oncology, Mekkah, Kingdom of Saudi Arabia, Email: abuzoooz60@gmail.com

\section{Introduction}

Small cell lung cancer (SCLC) is an aggressive disease that is virtually associated with smoking habits [1]. It contributes to $13 \%$ of lung cancer cases in USA [2]. In Europe males has higher incidence in Central/eastern part, whereas female incidence is noticed more in Northern part. In spite of the huge development in management of advanced non small cell lung cancer over the last few years including new molecular targeted therapies that help tailoring treatment in this disease as well as improving its outcome, there is no much progress has been done in clinical research of SCLC [3]. The standard management remains the same over the last 3 decades. This could be attributed to the lack of translational research in the field of SCLC. In SCLC there is limited tumor tissue for molecular studies since surgical treatment is rarely a standard treatment in this disease. This, in addition to the rapid disease progression with poor understanding of the mechanisms contributing to therapeutic resistance could be significant barriers for progress in SCLC's translational, clinical research and management [4].

SCLC is sensitive to both chemotherapy and radiation therapy. Complete response could be achieved in $40 \%$ to $60 \%$ in limited stage disease. Despite the high rate of response, median survival ranges from 12 to 20 months, with only $6 \%$ to $12 \%$ of patients are living beyond 5 years. Less than $5 \%$ of extensive stage SCLC lives more than2 years [5]. The demand for more translational research is obvious. Exploration of effective molecular targets may help in improving the dismal outcome of this disease. Clinicians use practical staging system for SCLC: limited disease (tumors limited to a single radiation portal) versus extensive disease (contra lateral hemi thorax or distant metastasis). However, the use of TNM staging can be advocated on the basis of its prognostic relevance [6]. Limited stage is usually treated with concomitant chemo radiation. In metastatic disease chemotherapy is the modality of treatment. Prophylactic cranial irradiation is used in both conditions in selected cases [7]. Not until recently therapeutic agents other than chemotherapy related to limited stage, relapsed or extensive stage SCLC started

Canc Therapy \& Oncol Int J 5(4): CTOIJ.MS.ID.555669 (2017) to be included in the in the current clinical practice guidelines $[7,8]$.

\section{Objectives}

To discuss the important cellular and molecular changes involved in the pathogenesis of SCLC as well as the pharmacological advances of targeted therapy in this disease, we did a literature review using unlimited midline search for the related topics.

Keywords: SCLC; Molecular biology; Molecular targets; Targeted therapy

\section{Results}

Small cell carcinomas are defined by their unique histology and are morphologically identical, regardless of the site of origin [9]. The morphological features of SCLC are so distinct that the diagnosis is based mostly on routine light microscopy. Cells express synaptophysin, chromogranin A and CD-56. Ancillary support by immune histo chemistry is only needed to rule out the limited differential considerations in special circumstances [9]. Two subtypes of SCLC are recognized: the pure form (70\%) and the combined form (30\%). Combined SCLC is defined as having a distinct non-small cell carcinoma component, such as adenocarcinoma, squamous cell carcinoma and large cell carcinoma [9]. A subset of NSCLCs with mutated EGFR return as SCLC when they are resistant to EGFR tyrosine kinase inhibitors [10]. A retrospective data based on 231 patients subgrouped patients of SCLC into peripheral or central according the primary location. In this study peripheral type was more common (56\%) Although it has been associated with higher frequency of interstitial lung disease, still peripheral type was an independent prognostic factor for better survival [11]

\section{Molecular Pathology}

\section{Tumor suppressors genes}

P53 protects against cellular genetic instability .Particularly it helps regulating cell cycle $\mathrm{G} 1 / \mathrm{S} \& 2 / \mathrm{M}$ transition by acting as 
transcription factor regulating downstream genes including p21, MDM2, GADD45 [12,13]. It is also involved in regulation of apoptosis by acting on $\mathrm{Bcl} 2$ associated $\mathrm{X}$ (BAX) which is a protein coding gene. P53 is located in chromosome 17p13. It is mutated in more than $90 \%$ of SCLCs [14]. Most inactivating mutations are point mutations in the DNA binding domain (missense mutation, 70\%-80\%), but homozygous deletions also occur [15]. P53 mutations correlate with cigarette smoking; in particular GC to TA trans- versions are caused by the carcinogen benzo (a) pyrene in tobacco smoke [16]. The MDM2 oncogene product inhibits p53 function by blocking its regulation of target genes and also enhances proteasome-dependent degradation of p53 [12].

\section{Retinoblastoma Gene (RB)}

Inactivation of both RB alleles at chromosome region 13q14 is common in lung cancers [17]. Protein abnormalities can be detected in about $90 \%$ of SCLCs [18]. Functional RB loss can include deletion, nonsense mutations, or splicing abnormalities which can frequently lead to a truncated RB protein [12]. The p16-cyclin D1-CDK4-RB pathway is involved in the cell cycle. CyclinD1 inhibit the activation of $\mathrm{RB}$ by stimulating its phosphorylation through CKD4. Hypophosphorylated RB controls transcription factors E2F1, E2F2 and E2 F3 which are necessary for G1/Stransition [19]. When RB is phosphorylated, E2F1 will be released and activated and that will allow cell cycle progression. Apoptotic protease activating factor -1(Apaf -1) andcaspases is proapoptoticgenes that can be suppressed by phosphorylated RB [20]. P16 is located in 9p21, it regulates RB through inhibition of CDK 4. Its mutation is less frequent in SCLC in comparison to NSCLC [12].

\section{3p Tumor suppressive Genes}

Allele loss involving chromosome arm $3 p$ is one of the most frequent (100\% in SCLC and 90\% in NSCLC) and earliest genetic alterations found in lung cancer [15]. 3 regions of $3 p$ loss where identified resulting in the presence of RASSFI A, FUSI1, SEMA3B, SEMA3F and FHIT/FRAB3 which are multiple tumor suppresser genes involved in cell cycle regulation as well as apoptotic mechanisms [21]. RAR beta gene which is a receptor for retinoic acid is also located in 3p24. It functions by methylation $(72 \%$ incidence in small cell lung cancer) [22].

\section{Transforming Growth Factor Beta}

Plays an important role in cell proliferation and survival. In the early stage of small cell cancer it inhibits cell proliferation. Later on, it conversely, induces angiogenesis [23].

\section{Ongogenes}

\section{MYCfamily}

Include 3protein c -MYC, n -MYC, L -MYC. Amplification of one member of the MYC family occurs in $18 \%$ to $31 \%$ of SCLCs [24]. c-MYC amplification occurs in both SCLC and NSCLC, whereas n-MYC and L-MYC amplifications nearly always occur in SCLC [15]. Bcl2 is expressed in more than $80 \%$ in SCLC [25]. $\mathrm{Bcl} 2$ is an important gene in regulation of apoptosis (inhibiting factor). It promotes cell survival and involved in tumor genesis as well resistance to chemotherapy [26].

\section{Growth promoting signaling pathways PI3K/AKT/PTEN Pathway}

PI3Ksarea group of protein kinases that regulates several cellular processes such as cell proliferation, survival, growth, apoptosis, cytoskeletal rearrangement (motility and adhesions) $[27,28]$. They activate the downstream pathway AKT and mTOR which regulates protein synthesis through targeting ribosomal protein S6 kinase 1 (S6K1) as well as eukaryotic Enhanced translation initiation factor $4 \mathrm{E}$-binding protein 1 (4EBP1).The tumor suppressor gene PTEN is a negative regulator of this pathway [29-31]. PI3K and PTEN mutations can be found in SCLC. Phosphorylated AKT is found in 70\% of SCLC cases. There is also elevation of phosphorylated4EBP1 and mTOR protein expression [32,33].

\section{RAS/RAF/MEK/ERK Pathway}

RAS mutation is usually found in NSCLC specially adenocarcinoma (20-30\%) but it is rare in SCLC [19]. ERBB1 and ERBB2 which are transmembrane kinase receptors, responsible for initiating cellular transduction signaling in MAP kinase pathway are also do not demonstrate abnormalities in SCLC in comparison to NSCLC $[15,19,34]$.

\section{Other tyrosine kinase receptors: cKIT}

SCLC cells express cancKIT receptor and its ligand stem cell factor in $40-60 \%[15,35,36]$. When binding to its ligand this receptor activate JAK- STAT, PI3K and MAP kinase pathways. This will initiate cell growth and differentiation [33].

\section{cMET}

Over expression and amplification of cMET was demonstrated in SCLC. This receptor is activated by its ligand hepatocye growth factor which will lead to downstream signaling of PI3K and STAT 3 pathway. This pathway regulates cell proliferation, survival, motility and ability of invasion $[27,29]$. Higher level of hepatocyte growth factors was associated with poor prognosis in this disease [27,33].

\section{Fibroblast Growth Factor}

one mechanism for the activation of PI3K-AKT and Ras/Raf/ MEK/Erk signaling pathways is through the binding of fibroblast growth factor (FGF) to fibroblast growth factor receptor (FGFR). Elevation ofFGF-2 an isoform of this tyrosine kinase is associated with chemo resistance and poor outcome in SCLC $[37,38]$. 


\section{nsulin like growth factor -1}

Insulin like growth factor -1 and insulin like growth factor 2 are ligands that bind to insulin growth like factor receptor-1, this will lead to activation of PI3K-AKT pathway in SCLC. IGF-1 is elevated in more than $95 \%$ of SCLC cell lines $[27,33]$.

\section{Angiogenesis}

Tumor angiogenesis is important for cancer cell survival, growth and invasion. It is controlled by several mechanisms like vascular endothelial growth factor (VEGF) which acts on VEGF receptors (VEGCF-R) high level of VEGF have been found in patients of small cell cancer [33]. Basic fibroblast growth factor also induces angiogenesis FGF-2) [12]. Chemokines (like interleukin-8) may act as angiogenic factors for small cell cancer [39].

\section{Developmental (cell fate) pathways}

\section{Notch pathway}

This pathway is important for regulation of airway epithelial development specially in determining neuroendocrine versus non-neuroendocrine differentiation [29,40]. Notch signaling is initiated by activation of Notch receptors (type 1-type 4) by 3 ligands (Delta 1, Jagged one and Jagged 2) [41]. This will further lead to activation of transcription targets such asHes1. In turn Hes1 will block transcription of human achaete-scute homolog-1 (h-ASH-1), which is important for the development of neuroendocrine cell in the lungs $[29,40]$. Notch-1 is in active in SCLC, howeverh-ASH-1 is highly expressed [40].

\subsection{Hedgehog pathway}

There are 3 ligands in this pathway: Indian (IHh), Sonic (SHh) and Desert (DHh) Hedgehog. Binding of Hh ligands to Patched receptor one (Ptch-1) with lead to release to release of Smotransmembrane protein which in turn activate the downstream transcription of $\mathrm{Hh}$ targets in the nucleus including GLi -1 [29]. In small cell lung carcinoma, the activation of this pathway could be one if the mechanism for chemotherapy resistance in case of presence of progenitor cell in SCLC [29,42]. Wht pathway is activated through Dishevelled (Dvl) over expression mainly in NSCLC [43].

\section{Role of Immunity}

Targeting body immune response rather than the cancer cell itself is becoming a rapidly evolving therapeutic approach. The role of immunity in solid malignant tumors has been observed through the presence of spontaneous regression in some cancers like melanoma and renal cell carcinoma. This approach is under investigation in SCLC.

$\mathrm{T}$ regulator cells has inhibitory effect under inflammatory conditions to prevent autoimmunity by either secreting substances like IL-10 and TGF beta or by expression of inhibitory receptors like programmed cell ligand - (PD-L1) or cytotoxic $\mathrm{T}$ lymphocyte associated antigen CTLA-1. Together with stimulatory molecules ( GITR, OX40, ICOS) regulated by binding of CD28 TO B7 in the antigen presenting cells, these molecules and receptors are called immune check points. Evading immune destruction is one of mechanism of the cancer cell to survive and develop as shown by Hanahan and Weinberg [44]. Immunediting is defined as multistep process including three phases: elimination of highly immugenic cancer cells, equilibrium between immunomedited destruction and adaptive immune system which will leave behind persistent weakly immugenic malignant clones/variants which will be able in the third phase to escape the immune system [44-46].

\section{Other mechanisms of SCLC Molecular Pathogenesis}

a. Telomerase enzyme: This enzyme is detected in $100 \%$ of cases of small cell cancer [47]. Up-regulation of telomerase maintains telomeric repeat and thus prevents shortening of cell life span [48].

b. Heat shock proteins-90: it as molecular chaperone that regulates the folding, stabilization and function of a number of proteins with important cellular functions [49]. It is a major inhibitor of apoptosis in SCLC [50].

c. Cell surface markers: CD56 is an isoform encoded by the NCAM gene (neural cell adhesion molecule) which modulates neuroendocrine cell growth, migration and differentiation. NCAM is found in $100 \%$ of SCLC. NCAM ligands or antibodies can inhibit malignant cell progression $[29,51]$.

d. Gangliosides: these are glycolipids, usually found in central nervous cell membranes. They interfere with adhesion molecules and cell receptors. FucGM1 (70\% expression), GM2 (60\% expression) and GD3 (405 expression) are types of gangliosides described in SCLC [52]. CD 24 is a cancer stem associated cell membrane protein involved in cell adhesion and can promote cell invasion. It is highly expressed in SCLC but rare in NSCLC $[53,54]$.

e. Proteomic and epigenetic changes: Poly ADP ribose polymerase 1 (PARP1) is found to be over- expressed in SCLC [55]. PARP inhibition down regulates key components of the homologous recombination pathway, such as RAD51 and BRCA1 [56]. Enhancer of zeste homolog 2(EZH2) is a protein crucial for SCLC cell survival. It also over expressed in SCLC $[57,58]$.

The role of targeting molecular alterations in treating SCLC

\section{Targeting tumor suppressive genes}

Mutated p53 has long half life and highly expressed in cancer cells which make it more amenable to cancer immunotherapy [29]. DC-ad- p53 is dendritic cell transduced with human wild type p53 containing recombinant adenovirus have been studied 
in SCLC in a phase I/ II study combined second chemotherapy and DC-ad-p53 improved survival in those who were evaluated to have positive immune response, however this was not statistically significant upon randomization in phase II $[59,60]$. Another study investigated the vaccine in 29 patients, showed similar results [61]. So far there are no clinical trials that show significant clinical benefit to this mode of treatment.

In regard to RB protein it was shown in a preclinical trial that agents like heat shock protein-90 inhibitor (17-AAG) can cause cell cycle arrest at G1 mediated by the presence of RB, however in cancer cell lacking RB proteins the cell cycle progress through G1,cells undergo arrest at M1 phase then become apoptotic but do not differentiate. Induction of differentiation by introduction of retinoblastoma gene in the cells my help increasing the antitumor effect of the drug [62]. Further investigation is needed in SCLC to explore the efficacy of this mechanism. Again, no significant results from clinical trials targeting $3 p$ tumors suppressive genes or RAR beta expressing tumors.

\section{Targeting Oncogenes}

Oblimeresan is an antisense oligo nucleotide molecule inhibiting bcl-2tested in SCLC results were promising in the preclinical studies but did show even worst outcome when it was combined with carboplatin and etoposide in phase II trial. Side effects were more in the experimental arm [63]. Another oral agent at-101(gossypol) was found to be inactive in recurrent chemo sensitive small cell lung carcinoma [64]. Obatodax mesyalate is an intravenous antiBcl2 failed to increase response when added to topotecan in phase II study [65]. In a preclinical study antisense MYCRNA expression has increased the sensitivity of platinum agents but not doxorubicin or vincristine, in chemotherapy -resistant SCLC cell lines [66]. In another preclinical study controlling MYC -eIF4E (eukaryotic translation initiation factor) by si RNA was associated with decrease resistance to everolimus when it was used to treat SCLC [67]. R-enolase is a bifunctional gene encoding a glycolytic enzyme and a DNA binding protein, c-MYC binding protein (MBP-1). MBP-1 binds the c-MYC promoter and down-regulates c-MYC transcription [53]. There is a study suggested that upregulation of these genes may inhibit c- MYC activity $[53,68]$. Targeting oncogenes needs further validated translational and clinical research to prove its efficacy in SCLC.

\section{Targeting signaling pathways}

PI3K/AKT/PTEN pathway: Inhibitors of this pathway have been tested in both preclinical and early phase clinical trials. mTOR inhibitor everolimus was used as single agent in small cell lung cancer patients who progress on chemotherapy but with limited efficacy [69]. A phase1b study combined everolimus with chemotherapy. The treatment was associated with increased incidence of infectious and hematological toxicities mandated limiting the dose of everolimus to $2.5 \mathrm{mg}$ and using GSCF support for all patients. The best overall response was partial response. With this data the authors concluded that everolimus is unlikely to be further investigated in an unselected SCLC population [70].

Temsirolimus is other intravenous mTOR inhibitors that did not show survival benefit as a maintenance treatment [71]. Dual inhibition of mTOR and other pathways was also tried as a treatment strategy; In a preclinical study there was as synergistic effects of erlotinib (EGFR inhibitor) and everolimus combination therapy at molecular level: cell viability, proliferation and autophagy [72]. mi RNA was also used to dysregulate the PI3K/ AKT/PTEN pathway $[56,73]$. Some authorities also tried dual pi3K and mTOR inhibition or Pan PI3k pathway inhibition in early phase clinical trials [29]. So far none of these trials has given enough clinical evidence to change the current clinical practice.

\section{EGFR}

As expected single agent EGFR mutation inhibitor gefitinib did not improve neither the survival nor the response in small cell cancer due to lack of expression of epidermal growth factor receptors in this disease [74]. Careful selection of patients may help some categories that could benefit from this strategy, since there is presence of histological subset of small cell cancer includes combined NSCLC and SCLC. Again third generation EGFR inhibitors may play a role since some EGFR resistant NSCLC may return back as small cell lung cancer, however further molecular studies is needed to identify the right patients.

\section{cKIT}

Imatinib which targetsc- kit receptors as well as platelet derivative growth factor receptors failed to show real objective response or survival benefit either in combination with chemotherapy carboplatin and etoposide or as maintenance after first line chemotherapy [75]. A phase II clinical trial tried high dose imatinib $400 \mathrm{mg}$ bid in patients who expressed c kit receptors but the results were not impressive [76].

\section{cMET}

A preclinical trial used small interfering RNA (siRNA) and selective prototype c-MET inhibitor SU11274, inhibiting the phosphorylation of c-MET itself and its downstream molecules such as AKT, S6 kinase, and ERK1 [77]. Multiple agents inhibiting c-MET were tried in early phase clinical trial without obvious effect. Ponatinibis ananti FGFR (fibroblast growth factor receptor) which is currently under investigation for both small and non SCLC (NCT01935336). Another study (NCT02109016) is recruiting patients to assess the efficacy of the FGFR-3inhibitor lucitanib.

\section{Insulin like growth factor}

Further translational and clinical research is needed to assess the efficacy of this target. For example AMG 479 is currently in clinical trial in combination with platinum and etoposide chemotherapy in SCLC [NCT00791154].

\section{Angiogenesis}


Bevacizumab a humanized monoclonal antibody targeting VEGFA receptor was investigated in several phase II trials. In view of promising results, a phase III clinical trial assigned patients to receive cisplatin plus etoposide ( $\operatorname{arm} A$ ) or the same regimen with bevacizumab ( $\mathrm{arm} B$ ) for a maximum of six courses. In the absence of progression, patients in arm B continued bevacizumab alone until disease progression or for a maximum of 18 courses. The primary end point was overall survival (OS). All patients included in the study were treatment naïve. The results were recently published, the addition of bevacizumab to cisplatin and etoposide in the first-line treatment of EDSCLC had an acceptable toxicity profile and led to a statistically significant improvement in progression-free survival $(\mathrm{P}=0.03)$, which, however, did not translate into a statistically significant increase in OS [78].

Sorafinib (acts against VEGFR-2, VEGFR-3) and sunitinib are small molecule multitargeted agents which were both investigated in extensive SCLC. So far, the results are not carrying anypractice changing data. Some of these studies are still ongoing. [NCT00466232, NCT00182689 for sorafinib\}.\{, NCT00616109, NCT00453154 for sunitinib\}. Aflibercept acts as VEGF trap by inhibiting the binding of VEGF-A, VEGF B to their receptors. This agent is still under investigation in SCLC in a phase II trial [NCT00828139\}.Some of the agents were found to act in part via inhibition of angiogenesis for example matrix metalloproteinase inhibitors and thalidomide. Both was investigated in SCLC and found to have no impact on survival. Actually there were associated with more side effects $[79,80]$.

\section{Targeting developmental (cell fate) pathways}

Although activation of Notch is suggested as away to suppress SCLC cells there no active agents that studied in phase clinical trial to prove this therapeutic strategy. However a preclinical study a DLL3(inhibitor of Notch pathway)targeted antibody-drug conjugate (ADC), showed efficacy in patient derived engrafts models [81]. Hedgehog(HH)pathways inhibitor vismodegib was investigated in small cell lung cancer in combination with first line chemotherapy but there was no clinical benefit(NCT00887159)[82]. Another agent so nidegib also showed activity against SCLC [83]. IPI-926 is a third agent targeting $\mathrm{HH}$ pathway which was tested in phase I trial including several types of cancer in patient who failed multiple lines of treatment [84]. As it was discussed before, Wnt pathway is mainly involved in non SCLC.

\section{Targeting immunotherapy}

Clinical research is expanding in this field. CTLA4 inhibitors, PD1/ PDL1 inhibitors and vaccines all investigated in SCLC. Ipilimumab(anti CTLA 4) has been tested in phase II study in combination with chemotherapy or after chemotherapy(phased regimen).The purpose of the phased regimen is to allow antigen release induced by chemotherapy before exposure to ipilimumab [85]. The phased regimen arm has significantly improved the immune related progression free survival (HR0.64, $\mathrm{p}=0.03$ ) but did not change the progression or the overall survival. there is an ongoing phase III clinical trial the purpose of this study is to determine whether the addition of ipilimumab to etoposide and platinum therapy will extend the lives of patients with ED SCLC more than etoposide and platinum therapy alone (NCT01450761).NivolumabaPD-1 inhibitor was investigated either alone or in combination to ipilimumab in a phase I/II trial (Checkmate 32). Nivolumab monotherapy and nivolumab plus ipilimumab showed antitumor activity with durable responses and manageable safety profiles in previously treated patients with SCLC [86]. (NCT02359019) is an ongoing phase II trial in which pembrolizumab has been given to extensive lung cancer patients after completion of chemotherapy.

Progression free survival is primary end point and overall survival will be a secondary one. Rovalpituzumab tsarina (ROVA -T) is an antibody-drug conjugate targeted to DLL3 on the surface of tumor cells. Since DLL3 is highly expressed in SCLC cells, the cytotoxic agent pyrrolo benzodiazepine will be delivered only to malignant cells rather than normal tissues with manageable toxicity [87]. Rovalpituzumab tsarina (ROVA -T) has demonstrated encouraging single-agent anti-tumor activity and durability in recurrent or refractory SCLC(NCT01901653). A phase II trial (NCT 02674568) investigating ROVA -T as third line or later treatment for subjects with relapsed or refractorydeltaLike protein 3-expressing SCLC is ongoing. Primary endpoints are objective response rate and overall survival. Although vaccine the rapies look promising from theoretical point of view, but they did not show real efficacy in treating SCLC $[61,88]$. The results of the randomized clinical study of maintenance therapy with immunomodulatory MGN1703 in patients with extensive disease SCLC after platinum-based first-line therapy are awaited (NCT02200081).MGN1703 activates the innate immune system via the Toll-like receptor 9 (TLR9) [89].

\section{Telomerase enzyme inhibitors}

Investigated in early phase clinical trials mainly in NSCLC [90]. Since this enzyme is highly expressed in SCLCas mentioned before, it is promising therapeutic target in the future.

\section{Targeting heat shock protein 90}

A Phase I/II studied ganetespib (a head shock protein 90 inhibitor) in combination with doxorubicin in solid tumors (Phase I). The results encouraged expanding this drug into phase II trial including refractory SCLC (NCT02261805). This trial was terminated in 2016 in view of negative results of ganetespib in a phase III study in non small cell carcinoma. An article published in The Journal of Expert Opinion in Investigational Drugs has evaluated ganetespib in both small and non small cell cancers. The authors' opinion is that ganetespib clinical trial was halted prematurely in SCLC despite preliminary evidence of prolonged stable disease in few patients [91].

\section{Targeting cell surface markers}

A phase II trial of huN901-DM1 in relapsed small cell cancer 


\section{Cancer therapy \& Oncology International Journal}

provided clinical activity and safety of this novel drug which is an immune conjugate that binds to the CD56 receptors internalize the cytotoxic agent DM1 [92]. Preclinical studies suggested that antiganglioside GD2 monoclonal anti bodies would be efficient when they are combined with other cytotoxic drugs [93]. Targeting CD24 through monoclonal antibodies SWA11 has shown some efficacy in preclinical trials. Immunoconjugation of doxorubicin with SW11 to introduce this cytotoxic drug inside the SCLC cells when it binds to cell surface CD24 was also investigated $[94,95]$.

\section{PARP-1 inhibitors}

Olaparib and talazoparib were used in SCLC. Some biomarkers has been found to predict positive response to talazoparib which are proteins involved in DNA damage Repair for example PARP,FANCD2 and pCHK2.0n the other hand high PI3K levels has predicted poor response to this drug $[55,96]$. Preclinical studies suggested thatEZH2 protein inhibition could be a potential target in treating SCLC [58].

\section{Statins}

It was shown that inhibition of -hydroxy-3-methylglutaryl coenzyme (HMG-CoA) reeducates, by stations may suppress the synthesis small G proteins such as RAS. In addition statins may induce pro-apoptotic, anti-angiogenic, and immunomodulatory effects [97]. When pervastatin was added to standard chemotherapy in small cell cancer in recent phase III study; the results were unsatisfactory [98].

\section{Discussion}

Despite the positive results in some preclinical research, the outcome of early phase trials as well as in the few phase III clinical trials done in this disease is disappointing so far. In view of the previously mentioned difficulties facing clinical research in SCLC, considering novel clinical trial designs may help overcoming these obstacles. Umbrella trial designs focus on molecular defined subsets of one histology type, each subset can test differ targeted agent [99]. On the other hand basket trial designs involve including multiple defined molecular subpopulations across different tumor types to evaluate the targeted therapy in question [99]. If a trial shows benefit from any targeted therapy under investigation, the predicted molecular feature can be potentially enriched. This is called adaptive enrichment designs [99]. The outcome from Molecular Screening for Optimization of Cancer Treatment (MOSCATO) 01 has recently reported higher progression free survival rates in hard- to- treat cancers when targeted therapy for different types of histology, including lung cancer which were selected using multigame panel test (NCT01566019). The difference is prognosis of small cancer when it is sub- typed according to location of the tumor might be an area for further translational research. Utilizing liquid biopsies may help understanding drug resistance in SCLC. In conclusion, the considerable number of molecular and genetic alternations which are present in small cell cancer has not been reflected in the management landscape of this disease. Changing research strategies might be a better way to evaluate the benefit of the current as well as new therapeutic agents in treating SCLC.

\section{References}

1. R Brownson, Chang J (1992) "Gender and histologic type variations in smoking-related risk of lung cancer." Epidemiology 3(1): 61-64.

2. R Govindan(2006) "Changing Epidemiology of Small-Cell Lung Cancer in the United States Over the Last 30 Years: Analysis of the Surveillance, Epidemiologic, and End Results Database," Journal of Clinical Oncology 24(28): 4539-4544.

3. A Jemal, Bray J, Ferlay (2011) "Global Cancer Statistics." CA Cancer J Clin 49(1): 33-64.

4. L A Byers, M Rudin(2015) "Small cell lung cancer: Where do we go from here," Cancer 664-672.

5. R Clark, Ihde(1998) "Small-cell lung cancer: treatment progress and prospects," Oncology 12( 5): 643-647.

6. E Vallières (2009) “The IASLC Lung Cancer Staging Project: Proposals Regarding the Relevance of TNM in the Pathologic Staging of Small Cell Lung Cancer in the Forthcoming (Seventh) Edition of the TNM Classification for Lung Cancer." J Thorac Oncol 4(9): 1049-1059.

7. M Früh D, De Ruysscher S, Popat L, Crinò (2013) “Small-cell lung cancer (SCLC): ESMO clinical practice guidelines for diagnosis, treatment and follow-up 24-6.

8. NCCN (2017) "NCCN clinical guidelines, small cell lung cancer".

9. M K Rezaei, N J Nolan, A M Schwartz(2013) "Surgical Pathology of Lung Cancer," Semin Respir Crit 34(6): 770-786.

10. M G Oser, Niederst, Sequist, Engelman (2015) "Transformation from non-small-cell lung cancer to small-cell lung cancer: Molecular drivers and cells of origin," The Lancet Oncology 16(4): 165-172.

11. N Kanaji (2017) "Peripheral-type small cell lung cancer is associated with better survival and higher frequency of interstitial lung disease." Lung Cancer108: 126-133.

12. M Kwun, M Fong MD (1999) “molecular pathogenesis of lung cancer," J Thorac Cardiovasc 118(6): 1136-1152.

13. R E Tamura, Vasconcellos D, Sarkar (2012) “GADD45 proteins: central players in tumor genesis" 12(5): 634-651.

14. Wistuba a, Gazdar, Minna (2001) “Molecular genetics of small cell lung carcinoma.," Seminars in oncology 28(4): 3-13.

15. M Sato, Shames, Gazdar, Minna(2007) "A Translational View of the Molecular Pathogenesis of Lung Cancer." J Thorac 2(43): 27-343.

16. M Sattler, R Salgia (2003) "Molecular and cellular biology of small cell lung cancer." Semin Oncol 30(1): 57-71.

17. Harbour, Shinn-Liang J, Whang-Peng (1988) "Abnormalities in structure and expression of the human retinoblastoma gene in SCLC". 241(4863): 353-370.

18. E Shimizu, Coxon A, Otterson GA, Steinberg SM, Kratzke RA (1994) “RB protein status and clinical correlation from 171 cell lines representing lung cancer, extrapulmonary small cell carcinoma, and mesothelioma". Oncogene 9(9): 2441-2448.

19. Y Seikdo, K M Fong, Minna (2007) "Molecular genetics of lung cancer" Annu 54: 73-87.

20. A Rodina "Selective compounds define Hsp90 as a major inhibitor of apoptosis in small-cell lung cancer." Nat Chem Biol 3(8): 498-507. 


\section{Cancer therapy \& Oncology International Journal}

21. K Huebner (2016) "Absence of FHIT Protein in Primary Lung Tumors and Cell Lines with FHIT Gene Abnormalities." Cancer Res 57(23): 5207-5212.

22. R- Gene (2000) "Promoter Methylation and Silencing of the Retinoic Acid AND". 92(16): 1303-1307.

23. M Dong and G, Blobe(2006) "Review in translational hematology-Role of transforming growth factor- in hematologic malignancies" Blood 107(12): 4589-4596.

24. DN Carney (1987) "The biology of lung cancer." Bulletin du Cancer 74(5): 495-500.

25. S Jiang, T Kameya, Y Sato (1996) "Bcl-2 Protein Expression in Lung Cancer and Close Correlation with Neuroendocrine Differentiation." Production 148(3): 837-846.

26. JM Adams, S Cory (2010) "The Bcl-2 apoptotic switch in cancer development and therapy." Oncogene 26(91): 1324-1337.

27. M Sattler, R Salgia (2003) “Molecular and cellular biology of small cell lung cancer." Seminars in Oncology 30(1): 57-71

28. L Sawyers (2002) "The phosphatidylinositol 3-Kinase-AKT pathway in human cancer." Nat Rev Cancer 2: 489-501.

29. D’Angelo, Pietanza (2010) “The molecular pathogenesis of small cell lung cancer." Cancer Biol 10: 1-10.

30. Liu Cheng, Roberts, Zhao (2009) "Targeting the phosphoinositide 3-kinase (PI3K) pathway in cancer" 8(8): 627-644.

31. Pal R, Figlin, Reckamp (2008) “The role of targeting mammalian target of rapamycin in lung cancer." 9(6): 340-345.

32. W Krystal, Sulanke, Litz (2002) "Inhibition of phosphatidylinositol 3-kinase-Akt signaling blocks growth, promotes apoptosis, and enhances sensitivity of small cell lung cancer cells to chemotherapy" Mol Cancer Ther 1(11): 913-922.

33. Fischer M, Marinov, Arcaro (2007) “Targeting receptor tyrosine kinase signalling in small cell lung cancer (SCLC): What have we learned so far" 33(4): 391-406.

34. Franklin, Veve, Hirsch, Helfrich, (2002)"Epidermal growth factor receptor family in lung cancer and premalignancy.", Seminars in oncology29(1): 43-14.

35. Cheng (2014), "Detection of c-kit mutational status in small-cell lung cancer in a Chinese cohort," Thorac Cancer 5(3): 225-231.

36. G Rossi (2003)"Kit Expression in Small Cell Carcinomas of the Lung : Effects of Chemotherapy," Clin Oncol 10(6): 1041-1047.

37. A Bikfalvi (2014) “Biological Roles of Fibroblast Growth Factor2 18(1): 26-45.

38. T Ruotsalainen, H Joensuu, K Mattson (2002) "High Pretreatment Serum Concentration of Basic Fibroblast Growth Factor Is a Predictor of Poor Prognosis in Small Cell Lung Cancer 11(11): 1492-1495.

39. D A Arenberg SL, Kunke PJ, Polverini M (1996 )“Inhibition of interleukin-8 reduces tumorigenesis of human non-small cell lung cancer in SCID mice." J Clin Invest 97(12): 2792-2802.

40. B J Collins, W Kleeberger, DW Ball (2004) "Notch in lung development and lung cancer," Seminars in Cancer Biology 14(5): 357-364.

41. C D Peacock, D N Watkins (2008) “Cancer stem cells and the ontogeny of lung cancer." J Clin Oncol 26(17): 2883-2889.

42. DN Watkins, DM Berman, SB Baylin (2003) "Progenitor Phenotype in Small-Cell Lung Cancer nd io sc ie nc fo is rib." Cell Cycle pp. 196-198.

43. K Uematsu, B He, L You, Z Xu, F McCormick (2003) "Activation of the Wnt pathway in non small cell lung cancer: evidence of dishevelled overexpression." Oncogene 22(46): 7218-7221.
44.D Hanahan, Weinberg (2011)“Hallmarks of cancer: The next generation" 144(5): 646-674.

45. M McPake (2010) ESMO: Handbook of Principles of Translational Research pp.19-24.

46. Teng, Swann C, Koebel RD, Schreiber(2008)"Immune-mediated dormancy: an equilibrium with cancer," J Leukoc Biol 84(4): 988-993.

47. K Kiyama (1995) "Telomerase activity in small-cell and non-small-cell lung cancers," J Natl Cancer Inst 87(12): 895-901.

48.JW Shay, WE Wright (2006) "Telomerase therapeutics for cancer: challenges and new directions," Nat. Rev. Drug Disco no 5(7): 577-584.

49. M Santarpia (2016) "Targeted drugs in small-cell lung cancer". Transl lung cancer Res 5(1): 51-70.

50. A Rodina (2007) "Selective compounds define Hsp90 as a major inhibitor of apoptosis in small-cell lung cancer." Nature chemical biology 3(8): 498-507.

51. M Jensen, F Berthold (2007) "Targeting the neural cell adhesion molecule in cancer," Cancer Lett 2(58): 19-21.

52.T Brezicka, B Bergman S, Olling (2000) "Reactivity of monoclonal antibodies with ganglioside antigens in human small cell lung cancer tissues," Lung Cancer 28(1): 29-36.

53. BA Teicher (2014) "Targets in small cell lung cancer." Biochem Pharmacol 87(2): 211-219.

54. Kristiansen, Sammar, Altevogt (2004) "Tumour biological aspects of CD24, a mucin-like adhesion molecule," Journal of Molecular Histology 35(3): 255-262.

55. B Wen, Lampe, Roberts, Atkins (2013) "Proteomic markers of DNA repair and PI3K pathway activation predict response to the PARP inhibitor BMN 673 in small cell lung cancer Robert," Clin Cancer Res 19(22): 1158-1178.

56. N Karachaliou, S Pilotto, Lazzari (2016) "Cellular and molecular biology of small cell lung cancer: an overview." Transl. Lung Cancer Res 5(1): 2-15.

57.JT Poirier (2015) "DNA methylation in small cell lung cancer defines distinct disease subtypes and correlates with high expression of EZH2," Oncogene 34(48): 5869-5878.

58. R Hubaux, K Thu, "EZH2 promotes E2F driven SCLC tumorigenesis through modulation of apoptosis and cell cycle regulation." J Thorac Oncol 8(8): 1102-1106.

59. T Ishida (1999) "Dendritic cells transduced with wild-type p53 gene elicit potent anti-tumour immune responses" Immunol 117(2): 244251.

60. SJ AuAlberto, A Chiappori, a dendritic cell-based p53 vaccine insmal cell lung cancer: observed association between immune response and enhanced chemotherapy effect." Expert Opin Biol Ther 10(6): 983-991.

61.S J Antonia(2006) "Combination of p53 cancer vaccine with chemotherapy in patients with extensive stage small cell lung cancer. Clin Cancer Res 12(3): 878-887.

62. PN Mu, M Srethapakdi, M Moasser (2001) "Inhibition of Heat Shock Protein 90 Function by Ansamycins Causes the Morphological and Functional Differentiation of Breast Cancer Cells 1. Cancer Res 61: 2945-2952.

63. R Kim, M Emi, K Tanabe(2004), "Therapeutic potential of antisense Bcl-2 as a chemosensitizer for cancer therapy." Cancer 101(11): 2491 2502 .

64. Baggstrom "A phase II study of AT-101 (Gossypol) in chemotherapysensitive recurrent extensive-stage small cell lung cancer." Thorac Oncol 6(10): 1757-1760. 


\section{Cancer therapy \& Oncology International Journal}

65. PK Paik, Charles M Rudin, Maria C Pietanza, Andrew Brown, Naiyer A Rizvi, et al. (2011) "A phase II study of obatoclax mesylate, a Bcl2 antagonist, plus topotecan in relapsed small cell lung cancer." Lung Cancer 74(3): 481-485.

66. RC Van Waardenburg, Meijer C, Burger H, Nooter K, De Vries EG, et al. (1997) "Effects of an inducible anti-sense c-myc gene transfer in a drug- resistant human small-cell-lung-carcinoma cell line." Int J Cancer 73(4): 544-550.

67. M Matsumoto, Seike M, Noro R, Soeno C, Sugano T, et al. (2015) “Control of the MYC-eIF4E axis plus mTOR inhibitor treatment in small cell lung cancer." BMC Cancer 15: 241.

68. KC Sedoris, SD Thomas, DM Miller (2010) “Hypoxia induces differential translation of enolase/MBP-1." BMC Cancer 10: 157.

69. Tarhini A, Kotsakis A, Gooding W, Shuai Y, Petro D, et al. (2008) "Phase II study of RAD001 (Everolimus ) in previously treated small cell lung cancer ( SCLC )." Clin Cancer Res 16(23): 5900-5907.

70. B Besse, Heist RS, Camdige DR, Beck JT, Schmid P, et al. (2014) “A phase ib dose-escalation study of everolimus combined with cisplatin and etoposide as first-line therapy in patients with extensive-stage smallcell lung cancer," Ann Oncol 25(2): 505-511.

71. KJ Pandya, Dahlberg S, Hidalgo M, Cohen RB, Lee MW et al. (2007) "A randomized, phase II trial of two dose levels of temsirolimus (CCI779) in patients with extensive-stage small-cell lung cancer who have responding or stable disease after induction chemotherapy: a trial of the Eastern Cooperative Oncology Group (E1500)," J Thorac Oncol 2(11): 1036-1041.

72. K Schmid, Z Bago-Horvath, W Berger, A Haitel, D Cejka et al. (2010) "Dual inhibition of EGFR and mTOR pathways in small cell lung cancer." Br J Cancer 103(5): 622-628.

73. S Umemura, Mimaki S, Tada S, Ishii G, Niho S, et al. (2014) “Therapeutic priority of the PI3K/AKT/mTOR pathway in small cell lung cancers as revealed by a comprehensive genomic analysis.," J Thorac Oncol 9(9): 1324-1331.

74. AM Moore, Einhorn LH, Estes D, Govindan R, Axelson J, et al. (2006) "Gefitinib in patients with chemo-sensitive and chemo-refractory relapsed small cell cancers: A Hoosier Oncology Group phase II trial," Lung Cancer 52(1): 93-97.

75. BE Johnson,Thomas Fischer, Berthold Fischer, Thierry Le Chevalier (2003) "Phase II study of imatinib in patients with small cell lung cancer." Clin Cancer Res 16(1): 5880-5887.

76. LM Krug, Crapanzano JP, Azzoli CG, Miller VA, Rizvi N, et al. (2005) "Imatinib mesylate lacks activity in small cell lung carcinoma expressing c-kit protein: A phase II clinical trial." Cancer 103(10) 2128-2131.

77. PC Ma, MS Tretiakova, V Nallasura, R Jagadeeswaran, AN Husain, et al. (2007) "Downstream signalling and specific inhibition of c-MET/HGF pathway in small cell lung cancer: implications for tumour invasion." Br J Cancer 97(3): 368-377.

78. M Tiseo, Luca Boni, F Ambrosio, Andrea Ardizzoni (2017) "Italian, Multicenter, Phase III, Randomized Study of Cisplatin Plus Etoposide With or Without Bevacizumab as First-Line Treatment in ExtensiveDisease Small-Cell Lung Cancer: The GOIRC-AIFA FARM6PMFJM Trial." J Clin Oncol 35(12).

79. SM Lee, Woll PJ, Rudd R, Ferry D, O' Brien M, et al. (2009) “Antiangiogenic therapy using thalidomide combined with chemotherapy in small cell lung cancer: A randomized, double-blind, placebo-controlled trial," J Natl Cancer Inst 101(15): 1049-1057.

80. FA Shepherd, Giaccone G, Seymour L, Debruyne C, Bezjak A, et al. (2002) "Prospective, Randomized, Double-Blind, Placebo-Controlled Trial of Marimastat After Response to First-Line Chemotherapy in Patients With Small-Cell Lung Cancer: A Trial of the National Cancer
Institute of Canada-Clinical Trials Group and the European Orga." J Clin Oncol 20(22): 4434-4439.

81. LR Saunders, Bankovich AJ, Anderson WC, Aujay MA, Bheddah S, et al. (2015) "A DLL3-targeted antibody-drug conjugate eradicates highgrade pulmonary neuroendocrine tumor-initiating cells in vivo HHS Public Access," Sci Transl Med 7(302): 136.

82. T Chandra Prakash Belani, Suzanne Eleanor Dahlberg, Charles M Rudin, Martin Fleisher, Helen X Chen, et al. (2013) Penn State Hershey Cancer Institute, Hershey, PA; Dana-Farber Cancer Institute, Boston, MA; Sidney Kim, "Three-arm randomized phase II study of cisplatin and etoposide (CE) versus CE with either vismodegib (V) or cixutumumab (Cx) for patients with extensive stage-small cell lung cancer (ES-SCLC) (ECOG 1508)." J Clin Oncol 7508.

83. Y Abe, N Tanaka (2016) "The Hedgehog Signaling Networks in Lung Cancer: The Mechanisms and Roles in Tumor Progression and Implications for Cancer Therapy." Biomed Res Int 2016: 1-11.

84. A Jimeno, G Weiss, W Miller (2013) "Phase I study of the Hedgehog pathway inhibitor IPI-926 in adult patients with solid tumors." Clin Cancer Res 19(10): 2766-2774.

85. M Reck, Igor Bondarenko, A Luft, TJ Lynch (2013) “Ipilimumab in combination with paclitaxel and carboplatin as first-line therapy in extensive disease-small-cell lungcancer: Results from a randomized, double-blind, multicenter phase 2 trial," Annals Oncology 24(1): 7583.

86. SJ Antonia (2016) "Nivolumab alone and nivolumab plus ipilimumab in recurrent small-cell lung cancer (CheckMate 032): a multicentre, open-label, phase 1/2 trial," The Lancet Oncology 17(7): 883-895.

87. CM Rudin, Pietanza MC, Bauer TM, Ready N, Glisson BS, et al. (2016) "Rovalpituzumab tesirine, a DLL3-targeted antibody-drug conjugate, in recurrent small-cell lung cancer: a first-in-human, first-in-class, open-label, phase 1 study." Lancet Oncol 18(1): 42-51.

88. LM Krug, Raghupathi G, Hood C, Kris MG, Miller VA, et al. (2004) "Vaccination of patients with small-cell lung cancer with synthetic fucosyl GM-1 conjugated to keyhole limpet hemocyanin," Clin Cancer Res 10(18): 6094-6100.

89. MR Weihrauch, Richly H, Becker HJ, Schmidth M, Hacker UT, etal. (2015) "Phase I clinical study of the toll-like receptor 9 agonist MGN1703 in patients with metastatic solid tumours. Eur J cancer 51(2): 146-156.

90. I Fernandez Garcia, C Ortiz de Solorzano, LM Montuenga (2008) "Telomeres and telomerase in lung cancer." J Thorac Oncol 3(10): $1085-1088$

91. DS Subramaniam, EA Warner, G Giaccone (2017) “Ganetespib for small cell lung cancer," Expert Opin Investig Drugs 26(1): 103-108.

92.J McCann (2007) "Phase II trial of huN901-DM1 in patients with relapsed small cell lung cancer (SCLC) and CD56-positive small cell carcinoma," J Clin Oncol 25(18): 18084.

93. S Yoshida, H Kawaguchi, S Sato, R Ueda, K Furukawa (2002) "An AntiGD2 Monoclonal Antibody Enhances Apoptotic Effects of Anti-cancer Drugs against Small Cell Lung Cancer Cells via JNK (c-Jun Terminal Kinase) Activation." Jpn J Cancer Res 93(7): 816-824.

94. AV Salnikov (2013) "Antibody targeting of CD24 efficiently retards growth and influences cytokine milieu in experimental carcinomas.," Br J Cancer 108(7): 1449-1459.

95. BA Froesch, RA Stahel, U Zangemeister Wittke (1996) "Preparation and functional evaluation of new doxorubicin immunoconjugates containing an acid-sensitive linker on small-cell lung cancer cells," Cancer Immunol Immunother 42(1): 55-63.

96.J Murai (2014) "Stereospecific PARP trapping by BMN 673 and comparison with olaparib and rucaparib," Mol Cancer Ther 13(2): 433-443. 
97. CK Natalia, G Vallianou, Alexandra Kostantinou, Marios Kougias (2014) "Statins and Cancer," Anticancer agents 14(5): 706-712.

98. MJ Seckl (2017) “Multicenter, Phase III, Randomized, Double-Blind, Placebo-Controlled Trial of Pravastatin Added to First-Line Standard Chemotherapy in Small-Cell Lung Cancer (LUNGSTAR)." J Clin Oncol 35(14): 1506-1514.
99. SJ Mandrekar, SE Dahlberg, R Simon (2015) “Improving Clinical Trial Efficiency: Thinking outside the Box." Am Soc Clin Oncol Educ Book 35: e141-e147.

Your next submission with Juniper Publishers will reach you the below assets

- Quality Editorial service

- Swift Peer Review

- Reprints availability

- E-prints Service

- Manuscript Podcast for convenient understanding

- Global attainment for your research

- Manuscript accessibility in different formats ( Pdf, E-pub, Full Text, Audio)

- Unceasing customer service

Track the below URL for one-step submission https://juniperpublishers.com/online-submission.php 